\title{
Stabilité des polynômes
}

\author{
par \\ NidAl Ali (Calais)
}

\section{Introduction}

DÉfinition 1. Soient $K$ un corps, $f(x) \in K[x]$ un polynôme irréductible; on pose $f_{1}(x)=f(x)$ et pour tout entier $m \geq 2, f_{m}(x)=\left(f_{m-1} \circ f\right)(x)$. On dit que $f$ est un polynôme stable sur $K$ si pour tout $m \geq 1, f_{m}(x)$ est irréductible sur $K$.

Dans [4], les auteurs donnent une méthode efficace pour tester la stabilité de presque tous les polynômes unitaires quadratiques. Dans [10], Odoni montre que le polynôme générique

$$
G\left(s_{1}, \ldots, s_{n}, x\right)=x^{n}-s_{1} x^{n-1}+\cdots+(-1)^{n} s_{n}
$$

est un polynôme stable dans $A\left[s_{1}, \ldots, s_{n}, x\right]$ où $A$ est un anneau intégralement clos et $s_{1}, \ldots, s_{n}$ sont des variables algébriquement indépendantes sur le corps des fractions de $A$. Dans [5], les auteurs montrent la stabilité de tout polynôme irréductible de la forme $x^{n}-c \in R[x]$ où $R$ est soit $\mathbb{Z}$, soit un anneau des polynômes à une variable sur $\mathbb{Z}$ ou sur un corps algébriquement clos.

Soient maintenant $K$ un corps de nombres de degré $n$, et $\left\{w_{1}, \ldots, w_{n}\right\}$ une base d'entiers de $K$. Soient $u_{1}, \ldots, u_{n}$ des variables algébriquement indépendantes sur $K, \xi=u_{1} w_{1}+\cdots+u_{n} w_{n}$ et $F\left(u_{1}, \ldots, u_{n}, x\right)=\operatorname{Irr}(\xi, L, x)$ où $L=\mathbb{Q}\left(u_{1}, \ldots, u_{n}\right)$. Le polynôme $F$ est à coefficients entiers, homogène et de degré $n$. (Voir Remarque 2.)

DÉfinition 2. On dira que $F\left(u_{1}, \ldots, u_{n}, x\right)$ est un polynôme générique des entiers de $K$.

Le polynôme $F\left(u_{1}, \ldots, u_{n}, x\right)$ est générique dans le sens où, en spécialisant $u_{1}, \ldots, u_{n}$ dans $\mathbb{Z}$, on obtient $F\left(u_{1}^{*}, \ldots, u_{n}^{*}, x\right)=\left[\operatorname{Irr}\left(\xi^{*}, \mathbb{Q}, x\right)\right]^{d}$ où $d=n / t$ avec $t=\left[\mathbb{Q}\left(\xi^{*}\right): \mathbb{Q}\right]$ et $\xi^{*}$ est un entier de $K$.

2000 Mathematics Subject Classification: 11R09, 11T06, 12E10.

Key words and phrases: polynomial, irreducibility, iteration, stability, inert prime, totally ramified. 
Une question importante, motivée par le résultat d'Odoni cité ci-dessus, est de savoir si le polynôme $F\left(u_{1}, \ldots, u_{n}, x\right)$ est stable sur $L$. Si tel est le cas, est-ce qu'il y a une infinité de spécialisations de $\left(u_{1}, \ldots, u_{n}\right)$ en $\left(u_{1}^{*}, \ldots, u_{n}^{*}\right)$ dans $\mathbb{Z}^{n}$ tels que $F\left(u_{1}^{*}, \ldots, u_{n}^{*}, x\right)$ est stable sur $\mathbb{Q}$ ? Les trois théorèmes qui suivent montrent que la réponse est positive si certaines conditions arithmétiques sur $K$ sont verifiées.

ThÉORÈme 1 . Soit $K=\mathbb{Q}(\theta)$ où $\theta$ est une racine dans $\overline{\mathbb{Q}}$ d'un polynôme irréductible dans $\mathbb{Z}[x]$ de la forme $f(x)=x^{n}-c$. Alors :

(i) Il existe une infinité d'entiers $\alpha$ de $K$ tels que le polynôme minimal de $\alpha$ est stable sur $\mathbb{Q}$.

(ii) Le polynôme générique des entiers de $K$ est stable sur $L$.

ThÉORÈme 2. Soit $K$ un corps de nombres de degré $n$ dans lequel il existe un nombre premier $p$ de $\mathbb{Z}$ totalement ramifié. Alors :

(i) Il existe une infinité d'entiers $\alpha$ de $K$ tels que le polynôme minimal de $\alpha$ est stable sur $\mathbb{Q}$.

(ii) Le polynôme générique des entiers de $K$ est stable sur $L$.

EXEMPLE 1. Ce théorème s'applique en particulier pour toute extension galoisienne $K / \mathbb{Q}$ de degré premier $n$ car tout nombre premier $p$ de $\mathbb{Z}$ ramifié dans $K$ est totalement ramifié.

Maintenant, est-ce que la stabilité du polynôme générique est vraie lorsque $K$ possède un nombre premier $p$ inerte ? On va montrer que cela est vraie si on suppose qu'une certaine propriété de stabilité sur $\mathbb{F}_{p}$ est verifiée.

DÉFINITION 3. Soient $n, e$ deux entiers naturels non nuls, et $p$ un nombre premier de $\mathbb{Z}$. On définit la propriété $S(n, p, e)$ par : il existe un polynôme unitaire, irréductible de degré $n$ et stable sur $\mathbb{F}_{p^{e}}$.

Il faut remarquer que cette propriété peut aussi bien être vraie que fausse. (Voir section 3.)

ThÉORÈme 3. Soit $K$ un corps de nombres de degré $n$ dans lequel il existe un nombre premier $p$ inerte. On suppose que $S(n, p, 1)$ est vraie. Alors :

(i) Il existe une infinité d'entiers $\alpha$ de $K$ tels que le polynôme minimal de $\alpha$ est stable sur $\mathbb{Q}$.

(ii) Le polynôme générique des entiers de $K$ est stable sur $L$.

Remarque 1 . Soit $K$ un corps de nombres de degré $n$ dans lequel il existe un nombre premier $p$ inerte. Alors d'après le Théorème de Tschebotaröw $([3$, Lemme 3]), il existe une infinité de nombres premiers $l$ inertes dans $K$. Il est possible que pour l'un des ces nombres $l, S(n, l, 1)$ soit veri- 
fiée. Cela signifie que l'hypothèse du Théorème 3 relative à $S(n, p, 1)$ est probablement superflue.

Les trois théorèmes précédents donnent la stabilité de $F$ sur $L$ sous certaines conditions arithmétiques, mais dans le cas général, i.e. pour un corps de nombres $K$ de degré $n$ quelconque, on ne sait pas si $F$ est stable ou non.

Cependant on montre dans la section 4 le résultat suivant:

Proposition 1. Le polynôme $F_{2}\left(u_{1}, \ldots, u_{n}, x\right)$ est irréductible sur $L$.

\section{Lemmes préliminaires}

DÉfinition 4. Soient $K$ un corps de nombres de degré $n, \theta$ un élément primitif de $K$ sur $\mathbb{Q}$, et $A$ son anneau des entiers. On définit l'indice de $\theta$ (noté $I(\theta)$ ) comme le cardinal de $A / \mathbb{Z}[\theta]$.

Lemme 1 ([7, Art. 96, p. 176]). Soient $K$ un corps de nombres de degrén, $A$ son anneau des entiers, et $p$ un nombre premier rationnel tel que sa décomposition dans A est donnée par $A p=\prod_{i=1}^{t} \mathcal{P}_{i}^{e_{i}}$. On suppose qu'il existe $r_{1}, \ldots, r_{s+1}$ dans $\mathbb{N}^{*}$ tels que $r_{1}+\cdots+r_{s+1}=t$ et

$$
\left\{\begin{array}{l}
\operatorname{deg}\left(\mathcal{P}_{1}\right)=\operatorname{deg}\left(\mathcal{P}_{2}\right)=\cdots=\operatorname{deg}\left(\mathcal{P}_{r_{1}}\right):=c_{1}, \\
\operatorname{deg}\left(\mathcal{P}_{r_{1}+1}\right)=\operatorname{deg}\left(\mathcal{P}_{r_{1}+2}\right)=\cdots=\operatorname{deg}\left(\mathcal{P}_{r_{2}}\right):=c_{2}, \\
\vdots \\
\operatorname{deg}\left(\mathcal{P}_{r_{s}+1}\right)=\operatorname{deg}\left(\mathcal{P}_{r_{s}+2}\right)=\cdots=\operatorname{deg}\left(\mathcal{P}_{r_{s+1}}\right):=c_{s+1} .
\end{array}\right.
$$

Alors pour que $p$ divise tous les indices $I(\theta)$ pour tout entier primitif $\theta$ de $K$ il faut et il suffit qu'il existe $j \in\{1, \ldots, s+1\}$ tel que le nombre des polynômes unitaires, irréductibles sur $\mathbb{F}_{p}$ de degré $c_{j}$ est strictement plus petit que $r_{j}$.

Lemme 2 ([7, Th. 1, p. 137]). Soient $K$ un corps de nombres de degré $n$, $A$ son anneau des entiers, $F\left(u_{1}, \ldots, u_{n}, x\right)$ le polynôme générique des entiers de $K$, et $p$ un nombre premier de $\mathbb{Z}$ tel que la décomposition de $p$ dans $A$ est donnée par $A p=\prod_{i=1}^{t} \mathcal{P}_{i}^{e_{i}}$. Alors

$$
F\left(u_{1}, \ldots, u_{n}, x\right)=\prod_{i=1}^{t} F_{i}\left(u_{1}, \ldots, u_{n}, x\right)^{e_{i}}+p G\left(u_{1}, \ldots, u_{n}, x\right)
$$

où les polynômes $F_{i}\left(u_{1}, \ldots, u_{n}, x\right)$ sont irréductibles sur $\mathbb{F}_{p}$, unitaires, et $G\left(u_{1}, \ldots, u_{n}, x\right)$ n'est pas divisible par aucun facteur $F_{i}\left(u_{1}, \ldots, u_{n}, x\right)$ dans $\mathbb{F}_{p}\left[u_{1}, \ldots, u_{n}, x\right]$.

Lemme 3 ([7, Art. 95, p. 172]). Soient $K$ un corps de nombres de degrén, $\theta$ un élément primitif de $K$ sur $\mathbb{Q}$, et $f(x)=\operatorname{Irr}(\theta, \mathbb{Q}, x)$. On suppose que 


$$
f(x)=\prod_{i=1}^{t} f_{i}(x)^{e_{i}}+p h(x)
$$

avec tous les $f_{i}(x)$ irréductibles sur $\mathbb{F}_{p}$ et $\operatorname{deg}(h)<\operatorname{deg}(f)$. Alors les deux conditions suivantes sont équivalentes :

(i) $p \mid I(\theta)$.

(ii) Il existe $i \in\{1, \ldots, t\}$ tel que $e_{i} \geq 2$ et $f_{i}(x) \mid h(x)$ dans $\mathbb{F}_{p}[x]$.

DÉFinition 5. Soient $f(x)=a_{n} x^{n}+a_{n-1} x^{n-1}+\cdots+a_{1} x+a_{0}$ un polynôme à coefficients entiers et $r \geq 1$ un entier premier à $n$. On dit que $f$ est un polynôme $p^{r}$-Eisenstein pour un certain nombre premier $p$ de $\mathbb{Z}$ si :

(i) $\vartheta_{p}\left(a_{0}\right)=r$.

(ii) $\vartheta_{p}\left(a_{j}\right) \geq r$ pour tout $j=1, \ldots, n-1$.

(iii) $\vartheta_{p}\left(a_{n}\right)=0$.

LEMmE 4. Soient $K$ un corps de nombres de degré $n, A$ son anneau des entiers, et $p$ un nombre premier de $\mathbb{Z}$. Alors les trois conditions suivantes sont équivalentes :

(i) $p$ est totalement ramifié dans $K$.

(ii) Il existe un polynôme $\phi(x) \in \mathbb{Z}[x]$ et une racine $\alpha \in \overline{\mathbb{Q}}$ de $\phi$ tels que $K=\mathbb{Q}(\alpha)$ et $\phi(x)$ est un polynôme p-Eisenstein.

(iii) Il existe un polynôme $\psi(x) \in \mathbb{Z}[x]$ et une racine $\beta \in \overline{\mathbb{Q}}$ de $\psi$ tels que $K=\mathbb{Q}(\beta)$ et $\psi(x)$ est un polynôme $p^{r}$-Eisenstein pour un certain entier $r$ premier à deg $\psi$.

Preuve. (i) $\Rightarrow($ ii). Soit $\mathcal{P}$ un idéal premier de $A$ au-dessus de $p$. Alors $A p=\mathcal{P}^{n}$ avec $\mathcal{P} \cap \mathbb{Z}=p$.

Supposons que $p \mid I(\gamma)$ pour tout entier primitif $\gamma$ de $K$. Alors d'après le Lemme $1\left(A p=\mathcal{P}^{n}, j=1, c_{j}=1\right)$ le nombre des polynômes unitaires irréductibles dans $\mathbb{F}_{p}[x]$ et de premier degré, qui est égale à $p$, est strictement plus petit que le nombre des idéaux $\mathcal{P}_{i}$ de degré $c_{j}$, qui est 1 , donc $p<1$, ce qui est absurde. Donc il existe au moins un entier primitif $\lambda$ de $K$ tel que $p \nmid I(\lambda)$.

Maintenant soit $\left\{w_{1}, \ldots, w_{n}\right\}$ une base d'entiers de $K, \xi=u_{1} w_{1}+\cdots+$ $u_{n} w_{n}, F\left(u_{1}, \ldots, u_{n}, x\right)$ le polynôme générique des entiers de $K$. Puisque $p$ est totalement ramifié dans $K$, d'après le Lemme 2 on a

$$
F\left(u_{1}, \ldots, u_{n}, x\right)=L\left(u_{1}, \ldots, u_{n}, x\right)^{n}+p G\left(u_{1}, \ldots, u_{n}, x\right)
$$

avec $L\left(u_{1}, \ldots, u_{n}, x\right)$ linéaire en $u_{1}, \ldots, u_{n}, x$ et ne divisant pas le polynôme $G\left(u_{1}, \ldots, u_{n}, x\right)$ dans $\mathbb{F}_{p}\left[u_{1}, \ldots, u_{n}, x\right]$.

Soit $\left(l_{1}, \ldots, l_{n}\right) \in \mathbb{Z}^{n}$ tel que $L\left(u_{1}, \ldots, u_{n}, x\right)=x+l_{1} u_{1}+\cdots+l_{n} u_{n}$ et soit $\left(u_{1}^{*}, \ldots, u_{n}^{*}\right) \in \mathbb{Z}^{n}$ tel que $\lambda=u_{1}^{*} w_{1}+\cdots+u_{n}^{*} w_{n}$. 
Soit $f(x)=\operatorname{Irr}(\lambda, \mathbb{Q}, x)$. Alors

$$
f(x)=F\left(u_{1}^{*}, \ldots, u_{n}^{*}, x\right)=(x+a)^{n}+p G\left(u_{1}^{*}, \ldots, u_{n}^{*}, x\right) ;
$$

ceci implique que $f(x)=(x+a)^{n}+p h(x)$ avec $h(x)=G\left(u_{1}^{*}, \ldots, u_{n}^{*}, x\right)$.

Puisque $p \nmid I(\lambda)$, le Lemme 3 montre que $(x+a) \nmid h(x)$ dans $\mathbb{F}_{p}[x]$. On pose $\phi(X)=f(X-a)=X^{n}+p h(X-a)$; il est facile de voir que $\phi(X)$ est un polynôme $p$-Eisenstein.

(ii) $\Rightarrow$ (iii). Évident.

(iii) $\Rightarrow$ (i). On pose $\psi(x)=x^{m}+b_{m-1} x^{m-1}+\cdots+b_{1} x+b_{0} \in \mathbb{Z}[x]$. Soient $\mathcal{P}$ un idéal premier de $A$ au-dessus de $p$ et $e$ son indice de ramification. Les deux inégalités: $[\mathbb{Q}(\beta): \mathbb{Q}]=n \leq m$ et $e \leq[\mathbb{Q}(\beta): \mathbb{Q}]([9$, Théorème 21 , p. 65]) montrent que $e \leq m$. On va montrer que $e=m$. En effet, on a

$$
\psi(\beta)=0=\beta^{m}+b_{m-1} \beta^{m-1}+\cdots+b_{1} \beta+b_{0} \equiv \beta^{m}(\bmod \mathcal{P}),
$$

donc $\vartheta_{\mathcal{P}}(\beta)>0$. On en déduit que pour tout $j \in\{1, \ldots, m-1\}$,

$$
\vartheta_{\mathcal{P}}\left(b_{j} \beta^{j}\right)=\vartheta_{\mathcal{P}}\left(b_{j}\right)+j \vartheta_{\mathcal{P}}(\beta)=e \vartheta_{p}\left(b_{j}\right)+j \vartheta_{\mathcal{P}}(\beta)>e r=\vartheta_{\mathcal{P}}\left(b_{0}\right) .
$$

Il s'ensuit que

$$
\begin{aligned}
\vartheta_{\mathcal{P}}\left(-b_{m-1} \beta^{m-1}-\cdots-\right. & \left.b_{1} \beta-b_{0}\right) \\
& =\inf \left\{\vartheta_{\mathcal{P}}\left(-b_{m-1} \beta^{m-1}-\cdots-b_{1} \beta\right), \vartheta_{\mathcal{P}}\left(b_{0}\right)\right\} \\
& =\vartheta_{\mathcal{P}}\left(b_{0}\right)=e r .
\end{aligned}
$$

Ceci implique que $m \vartheta_{\mathcal{P}}(\beta)=e r$.

Puisque $\beta^{m}=-b_{m-1} \beta^{m-1}-\cdots-b_{1} \beta-b_{0}$, on a , $\vartheta_{\mathcal{P}}\left(\beta^{m}\right)=m \vartheta_{\mathcal{P}}(\beta)=e r$, donc $m \mid e r$. Comme $(m, r)=1$, on obtient que $m \mid e$ et par suite $m \leq e$, d'où l'égalité.

Corollaire 1. Soient $p$ un nombre premier de $\mathbb{Z}$ et $f(x)=a_{n} x^{n}+$ $a_{n-1} x^{n-1}+\cdots+a_{1} x+a_{0} \in \mathbb{Z}[x]$ un polynôme $p^{r}$-Eisenstein pour un certain entier naturel $r$ premier à $n$. Alors $f(x)$ est stable sur $\mathbb{Q}$.

Preuve. Dans (iii) $\Rightarrow(\mathrm{i})$, on a montré que $\operatorname{deg}(\psi(x))=[K: \mathbb{Q}]$, donc on obtient en même temps l'irréductibilité sur $\mathbb{Q}$ du polynôme $\psi(x)$ qui est $p^{r}$-Eisenstein. Ce résultat a été montré par Dumas ([6, 12-2, p. 252]) en utilisant le polygone de Newton.

Pour montrer qu'un polynôme $p^{r}$-Eisenstein est stable il suffit de montrer que le composé de deux polynômes $p^{r}$-Eisenstein est $p^{r}$-Eisenstein. En effet, soient $f(x)=a_{n} x^{n}+a_{n-1} x^{n-1}+\cdots+a_{1} x+a_{0}$ et $g(x)=b_{m} x^{m}+b_{m-1} x^{m-1}+$ $\cdots+b_{1} x+b_{0}$ deux polynômes à coefficients entiers, $p^{r}$-Eisenstein. On pose

$$
f \circ g(x)=c_{l} x^{l}+c_{l-1} x^{l-1}+\cdots+c_{1} x+c_{0}
$$

avec $l=m n, c_{l}=a_{n} b_{m}^{n}$ et

$$
c_{0}=f \circ g(0)=f\left(b_{0}\right)=a_{n} b_{0}^{n}+a_{n-1} b_{0}^{n-1}+\cdots+a_{1} b_{0}+a_{0} .
$$


On a $f(x) \equiv a_{n} x^{n}\left(\bmod p^{r}\right)$ et $g(x) \equiv b_{m} x^{m}\left(\bmod p^{r}\right)$, donc $f \circ g(x) \equiv c_{l} x^{l}$ $\left(\bmod p^{r}\right)$, d'où $p^{r}$ divise $c_{0}, \ldots, c_{l-1}$. Il est évident que $\vartheta_{p}\left(c_{0}\right)=\vartheta_{p}\left(a_{0}\right)=r$, $p \nmid c_{l}$ et que $(r, l)=1$, d'où le résultat.

Lemme 5 ([4, Théorème 5]). Soient p un nombre premier impaire de $\mathbb{Z}$, $q$ une puissance de $p$, et $f(x)=x^{2}-a x+b \in \mathbb{F}_{q}[x]$ un polynôme irréductible de discriminant d. Alors les deux conditions suivantes sont équivalentes:

(i) $f(x)$ est stable sur $\mathbb{F}_{q}$.

(ii) Pour tout entier $m \geq 1, f_{m}(-d / 4)$ n'est pas un carré dans $\mathbb{F}_{q}$.

LEMme 6 ([5, Corollaire 5]). Soit $f(x)=x^{n}-c \in R[x]$ un polynôme irréductible où $R$ est soit $\mathbb{Z}$, soit un anneau des polynômes à une variable sur $\mathbb{Z}$ ou sur un corps algébriquement clos. Alors $f$ est un polynôme stable dans $R[x]$.

LEMme 7 ([8, Théorème 16 , p. 221]). Soit $K$ un corps et $f(x)=x^{n}-\alpha$ $\in K[x]$. Alors $f(x)$ est réductible sur $K$ si et seulement si il existe p premier tel que $p \mid n$ et $\alpha \in K^{p}$, ou $4 \mid n$ et $\alpha \in-4 K^{4}$.

LEMmE 8. Soit $K$ un corps, $f(x), g(x)$ deux polynômes dans $K[x]$, et soit $\alpha$ une racine quelconque de $f(x)$ dans une clôture algébrique de $K$. Alors les deux conditions suivantes sont équivalentes.

(i) $f \circ g(x)$ est irréductible sur $K$.

(ii) $f(x)$ est irréductible sur $K$ et $g(x)-\alpha$ est irréductible sur $K(\alpha)$.

Preuve. Voir [11, Satz 4, p. 288] ou [1, énoncé 2.9] pour deux preuves différentes.

3. Stabilité sur les corps finis. Maintenant on va étudier la propriété $S(n, p, e)$ définie dans l'introduction, qui va intervenir dans le Théorème 3 . Tout d'abord il faut remarquer que $S(2,2,1)$ n'est pas vraie. En effet, le seul polynôme unitaire et irréductible du second degré sur $\mathbb{F}_{2}$ est $f(x)=x^{2}+x+1$ et il est instable puisque

$$
f_{3}(x)=\left(x^{4}+x^{3}+1\right)\left(x^{4}+x^{3}+x^{2}+x+1\right) .
$$

Proposition 2. Soient $p$ un nombre premier de $\mathbb{Z}$, et $n$, e deux entiers naturels non nuls. Alors :

(i) $S(n, p, e) \Rightarrow S(n, p, e \times s)$, pour tout entier $s$ premier à $n$.

(ii) $S(n, p, e) \Rightarrow S\left(n^{m}, p, e\right)$, pour tout entier $m \geq 1$.

Preuve. (i) Soient $s$ un entier premier à $n$ et $f(x)$ un polynôme unitaire, de degré $n$ et stable sur $\mathbb{F}_{p^{e}}$. Pour tout entier $m \geq 1$, on désigne par $\alpha_{m}$ l'une des racines de $f_{m}(x)$, donc $\left[\mathbb{F}_{p^{e}}\left(\alpha_{m}\right): \mathbb{F}_{p^{e}}\right]=n^{m}$. On considère le diagramme suivant : 


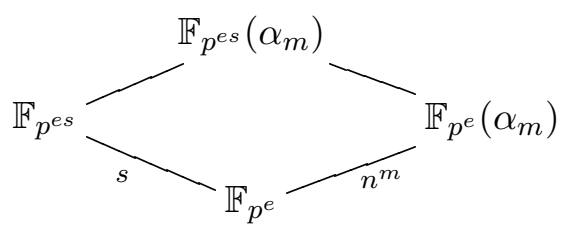

Comme $(s, n)=1$, on a $\left(s, n^{m}\right)=1$. Ceci implique que $\mathbb{F}_{p^{e s}}$ et $\mathbb{F}_{p^{e}}\left(\alpha_{m}\right)$ sont linéairement disjoints sur $\mathbb{F}_{p^{e}}$, donc $\left[\mathbb{F}_{p^{e s}}\left(\alpha_{m}\right): \mathbb{F}_{p^{e s}}\right]=\left[\mathbb{F}_{p^{e}}\left(\alpha_{m}\right): \mathbb{F}_{p^{e}}\right]=$ $n^{m}$; ainsi $f_{m}(x)$ est irréductible sur $\mathbb{F}_{p^{e s}}$ et par suite $f(x)$ est stable sur $\mathbb{F}_{p^{e s}}$.

(ii) Supposons que $S(n, p, e)$ est vraie. Il existe donc un polynôme $g(x)$ unitaire, irréductible de degré $n$, stable sur $\mathbb{F}_{p^{e}}$. Mais la stabilité de $g(x)$ implique celle de $g_{m}(x)$ qui a comme degré $n^{m}$; donc pour tout $m \geq 1$, $S\left(n^{m}, p, e\right)$ est vraie.

Proposition 3. Soit $p$ un nombre premier impair. Alors pour tout entier $e \geq 1, S(2, p, e)$ est vraie.

Preuve. On pose $q=p^{e}$ et on va montrer en utilisant le Lemme 5 qu'il existe un polynôme unitaire quadratique stable sur $\mathbb{F}_{q}$. Pour $a \in \mathbb{F}_{q}^{*}$ soit $\left(\frac{a}{q}\right)$ le symbole de Legendre; on note que $\left(\frac{a}{q}\right)=1$ si $a$ est un carré de $\mathbb{F}_{q}^{*}$ et $\left(\frac{a}{q}\right)=-1$ sinon.

On distingue deux cas selon les valeurs de $q$ :

1) $q \equiv 1(\bmod 4)$. Soit $a \in \mathbb{F}_{q}^{*}$ tel que $\left(\frac{a}{q}\right)=-1$; alors le polynôme $f(x)=(x-a)^{2}+a$ est stable sur $\mathbb{F}_{q}$. En effet, soit $d$ le discriminant de $f$; alors $d=-4 a$. Comme $\left(\frac{a}{q}\right)=-1$ et $\left(\frac{-1}{q}\right)=1$, on a $\left(\frac{d}{q}\right)=-1$, donc $f(x)$ est irréductible sur $\mathbb{F}_{q}$.

Puisque $-d / 4=a$ et $f(a)=a$, on a $f_{m}(a)=a$ pour tout $m \geq 1$, par suite le Lemme 5 s'applique et $f$ est stable sur $\mathbb{F}_{q}$.

2) Si $q \equiv-1(\bmod 4)$ alors $\left(\frac{-1}{q}\right)=-1$ et il existe $u, v \in \mathbb{F}_{q}^{*}$ tels que $-1=u^{2}+v^{2}([2$, énoncé $12-2])$.

Posons $f(x)=\left(x-4 u^{2}-2\right)^{2}+4 u^{2}$. Le polynôme $f$ est irréductible sur $\mathbb{F}_{q}$ car son discriminant $d=-16 u^{2}$ n'est pas un carré dans $\mathbb{F}_{q}$. Maintenant $-d / 4=4 u^{2}, f(-d / 4)=f\left(4 u^{2}\right)=-4 v^{2}$ et $f_{2}(-d / 4)=f\left(-4 v^{2}\right)=-4 v^{2}$. Or $-4 v^{2}$ n'est pas un carré dans $\mathbb{F}_{q}$, ce qui implique que pour tout $m \geq 1$, $f_{m}(-d / 4)$ n'est pas un carré dans $\mathbb{F}_{q}$ et par suite le polynôme $f(x)$ est stable $\operatorname{sur} \mathbb{F}_{q}$.

\section{Preuves des résultats}

Preuve du Théorème 1. (i) D'après le Lemme 6, le polynôme $f(x)=$ $\operatorname{Irr}(\theta, \mathbb{Q}, x)$ est stable sur $\mathbb{Q}$.

Maintenant pour tout $(i, l) \in \mathbb{Z}^{2}$ tel que $l$ est premier à $n$, on pose $\alpha_{i}=i \theta^{l}, g_{i}(x)=x^{n}-i^{n} c^{l}$; alors $g_{i}\left(\alpha_{i}\right)=0$. Le polynôme $g_{i}(x)$ est encore 
irréductible sur $\mathbb{Q}$. En effet, supposons que $g_{i}$ soit réductible sur $\mathbb{Q}$; ceci implique d'après le Lemme 7 qu'il existe un nombre premier $p$ divisant $n$ tel que $i^{n} c^{l} \in \mathbb{Q}^{p}$, ou $4 \mid n$ et $i^{n} c^{l} \in-4 \mathbb{Q}^{4}$.

Si $i^{n} c^{l} \in \mathbb{Q}^{p}$ alors $c^{l} \in \mathbb{Q}^{p}$. Or $(l, n)=1$, donc $c \in \mathbb{Q}^{p}$, d'où $f$ est réductible sur $\mathbb{Q}$, ce qui est absurde.

Si $4 \mid n$ et $i^{n} c^{l} \in-4 \mathbb{Q}^{4}$ alors il existe $a \in \mathbb{Q}$ tel que $c^{l}=-4 a^{4}$. On pose $c=-2^{h} d$ avec $d$ impair ; alors $c^{l}=-2^{h l} d^{l}=-4 a^{4}$. Pour tout nombre premier $q$ tel que $q \mid d$, on a $\vartheta_{q}(d) \equiv 0(\bmod 4)$, donc il existe $e \in \mathbb{Q}$ tel que $d=e^{4}$. Ceci implique que $c^{l}=-2^{h l} e^{4 l}=-4 a^{4}$, ce qui donne $h l \equiv 2$ $(\bmod 4)$, d'où $h \equiv 2(\bmod 4)$ puisque $l$ est impair. Par suite $c \in-4 \mathbb{Q}^{4}$, i.e. $f$ est réductible sur $\mathbb{Q}$, ce qui n'est pas possible. Donc $g_{i}$ est irréductible $\operatorname{sur} \mathbb{Q}$.

Ainsi on obtient une infinité d'entiers de $K$ ayant des polynômes minimaux stables sur $\mathbb{Q}$.

(ii) Soit $\alpha$ un entier de $K$ tel que $f(x)=\operatorname{Irr}(\alpha, \mathbb{Q}, x)$ est stable sur $\mathbb{Q}$. Alors il existe $\left(u_{1}^{*}, \ldots, u_{n}^{*}\right) \in \mathbb{Z}^{n}$ tel que $f(x)=F\left(u_{1}^{*}, \ldots, u_{n}^{*}, x\right)$, donc $F\left(u_{1}, \ldots, u_{n}, x\right)$ est stable sur $L$.

Preuve du Théorème 2. (i) Soit $p$ un premier de $\mathbb{Z}$ totalement ramifié dans $K$. D'après le Lemme 4 , on sait qu'il existe un entier primitif $\alpha$ de $K$ tel que $\phi(X)=\operatorname{Irr}(\alpha, K, x)$ est un polynôme $p$-Eisenstein. Soit $A$ l'anneau des entiers de $K$ et pour tout $i \in \mathbb{Z}$, soit $\alpha_{i}=\alpha+i p \in A$.

On peut vérifier que le polynôme minimal de $\alpha_{i}$ est encore $p$-Eisenstein, donc stable sur $\mathbb{Q}$. Ainsi on a construit une infinité d'entiers de $K$ ayant des polynômes minimaux stables sur $\mathbb{Q}$.

(ii) On fait le même raisonnement qu'en cas du Théorème 1.

Preuve du Théorème 3. (i) Soient $A$ l'anneau des entiers de $K$ et $g(x)$ le polynôme unitaire, de degré $n$ et stable sur $\mathbb{F}_{p}$. Comme $p$ est inerte, ceci implique que $A / p A$ est une extension de $\mathbb{F}_{p}$ de degré $n$. Soit $\beta \in A$ tel que $\bar{\beta}$ est un élément primitif de $A / p A$ et $\operatorname{Irr}\left(\bar{\beta}, \mathbb{F}_{p}, x\right)=g(x)$. Le polynôme $g(x)$ est stable sur $\mathbb{F}_{p}$, donc $\operatorname{Irr}\left(\bar{\beta}, \mathbb{F}_{p}, x\right)$ est stable sur $\mathbb{F}_{p}$ et par suite $\operatorname{Irr}(\beta, \mathbb{Q}, x)$ est stable sur $\mathbb{Q}$.

Pour tout élément $a \in A$ et pour tout entier $l \geq 0$, soit $\alpha=\beta^{p^{l}}+p a$; alors $\bar{\alpha}=\bar{\beta}^{p^{l}}$, donc $\operatorname{Irr}(\alpha, \mathbb{Q}, x)$ est stable sur $\mathbb{Q}$. Par conséquent, on a construit une infinité d'éléments de $A$ ayant des polynômes minimaux stables sur $\mathbb{Q}$.

(ii) On fait le même raisonnement qu'en cas du Théorème 1.

Remarque 2. Soient $K$ un corps de nombres de degré $n$, et $\left\{w_{1}, \ldots, w_{n}\right\}$ une base d'entiers de $K$. Soit $F\left(u_{1}, \ldots, u_{n}, x\right)=\operatorname{Irr}(\xi, L, x)$ le polynôme générique des entiers de $K$. Alors on a

$$
F\left(u_{1}, \ldots, u_{n}, x\right)=\prod_{i=1}^{n}\left(x-\xi_{\sigma_{i}}\right)
$$


où $\sigma_{1}, \ldots, \sigma_{n}$ désignent les $n$ plongements distincts de $K$ dans $\overline{\mathbb{Q}}\left(u_{1}, \ldots, u_{n}\right)$ et $\xi_{\sigma_{i}}=u_{1} \sigma_{i}\left(w_{1}\right)+\cdots+u_{n} \sigma_{i}\left(w_{n}\right)$. En effet, $\xi \in \mathbb{Q}\left(u_{1}, \ldots, u_{n}, w_{1}, \ldots, w_{n}\right)$ et $\left[\mathbb{Q}\left(u_{1}, \ldots, u_{n}, w_{1}, \ldots, w_{n}\right): \mathbb{Q}\left(u_{1}, \ldots, u_{n}\right)\right]=n$. Comme $\xi_{\sigma_{i}} \neq \xi_{\sigma_{j}}$ pour tout $i \neq j$, il s'ensuit que $\xi$ est un élément primitif de $\mathbb{Q}\left(u_{1}, \ldots, u_{n}, w_{1}, \ldots, w_{n}\right)$ sur $\mathbb{Q}\left(u_{1}, \ldots, u_{n}\right)$, donc $\operatorname{deg}_{x}(F(x))=n$. On a aussi montré que si $\theta$ est un élément primitif de $K$ alors $\mathbb{Q}\left(u_{1}, \ldots, u_{n}, \xi\right)=\mathbb{Q}\left(u_{1}, \ldots, u_{n}, \theta\right)$.

La formule de décomposition de $F$ montre en particulier que $F$ est homogène en $\left(u_{1}, \ldots, u_{n}, x\right)$.

Preuve de la Proposition 1. Supposons que $F_{2}\left(u_{1}, \ldots, u_{n}, x\right)$ est réductible sur $L$. Alors d'après le Lemme $8, F\left(u_{1}, \ldots, u_{n}, x\right)-\xi$ est réductible sur $L(\xi)$. Mais d'après la Remarque $2, L(\xi)=L(\theta)=\mathbb{Q}\left(\theta, u_{1}, \ldots, u_{n}\right)$, donc soit

$$
\begin{aligned}
F\left(u_{1}, \ldots, u_{n}, x\right)-\xi= & T\left(u_{1}, \ldots, u_{n}, x\right) \cdot H\left(u_{1}, \ldots, u_{n}, x\right) \\
= & {\left[T_{m}(*)+T_{m-1}(*)+\cdots+T_{1}(*)+T_{0}(*)\right] } \\
& \cdot\left[H_{k}(*)+H_{k-1}(*)+\cdots+H_{1}(*)+H_{0}(*)\right]
\end{aligned}
$$

avec $m+k=n, T_{i}$ et $H_{j}$ désignant les composantes homogènes de $T$ et $H$ de degrés respectifs $i$ et $j$ dans $\mathbb{Q}\left(\theta, u_{1}, \ldots, u_{n}\right)[x]$. Pour faciliter l'écriture on a noté $\left(u_{1}, \ldots, u_{n}, x\right)=(*)$. Or le polynôme $F(*)$ est homogène, donc on en déduit après identification que

$$
\begin{array}{ll}
(1) & T_{m}(*) H_{k}(*)=F(*), \\
(2) & T_{m}(*) H_{k-1}(*)+T_{m-1}(*) H_{k}(*)=0, \\
(3) & T_{m}(*) H_{k-2}(*)+T_{m-1}(*) H_{k-1}(*)+T_{m-2}(*) H_{k}(*)=0, \\
& \vdots \\
(n) & T_{1}(*) H_{0}(*)+T_{0}(*) H_{1}(*)=-\xi, \\
(n+1) & T_{0}(*) H_{0}(*)=0 .
\end{array}
$$

Comme $F(*)$ est irréductible sur $L=\mathbb{Q}\left(u_{1}, \ldots, u_{n}\right)$, il est séparable. Ceci implique que d'après $(1), T_{m}(*)$ est premier à $H_{k}(*)$ dans $L(\theta)[x]$. Or d'après (2), $T_{m}(*) \mid T_{m-1}(*) \cdot H_{k}(*)$, donc $T_{m}(*) \mid T_{m-1}(*)$. La seule possibilité est que $T_{m-1}(*)=0$. De même on montre que $H_{k-1}(*)=0$. Maintenant l'équation (3) s'écrit $T_{m}(*) H_{k-2}(*)+T_{m-2}(*) H_{k}(*)=0$. Ceci implique que $T_{m}(*) \mid T_{m-2}(*)$ et $H_{k}(*) \mid H_{k-2}(*)$, donc $T_{m-2}(*)=H_{k-2}(*)=0$ et par suite on obtient avec le même raisonnement que $T_{i}(*)=0, H_{j}(*)=0$ pour tout $(i, j) \in\{2, \ldots, m-1\} \times\{2, \ldots, k-1\}$. Puisque $T_{0}(*) H_{0}(*)=0$, on peut supposer que $T_{0}(*)=0$.

La formule $(n)$ montre que $T_{1}(*) H_{0}(*)=-\xi$. On pose $H_{0}(*)=b$ avec $b \in Q(\theta)$; donc il existe $a \in Q(\theta)$ tel que $T_{1}(*)=a \xi$ et $a b=-1$. Par suite on obtient

$$
F(*)-\xi=\left[T_{m}(*)+a \xi\right]\left[H_{k}(*)+H_{1}(*)+b\right] .
$$


On a $H_{1}(*)=0$, sinon dans $F(*)-\xi$, il y aurait une composante homogène de degré 2 qui est $a \xi H_{1}(*)$, ce qui n'est pas possible. Donc $F(*)-\xi=$ $\left[T_{m}(*)+a \xi\right]\left[H_{k}(*)+b\right]=T_{m}(*) H_{k}(*)+b T_{m}(*)+a \xi H_{k}(*)+a b \xi$. Après identification des parties homogènes de deux membres de l'égalité on obtient $b T_{m}(*)+a \xi H_{k}(*)=0$, ce qui est absurde puisque $a$ et $b$ sont non nuls et $T_{m}(*)$ est premier à $H_{k}(*)$ dans $L(\theta)[x]$.

5. Conclusion. Dans les trois théorèmes, on a montré qu'il existe une infinité d'entiers du corps $K$ tels que leurs polynômes minimaux sont stables sur $\mathbb{Q}$, et aussi la stabilité sur $L$ du polynôme générique des entiers $F\left(u_{1}, \ldots, u_{n}, x\right)$. Mais il faut remarquer que la stabilité de $F$ sur $L$ n'implique pas en général l'existence d'un ou plusieurs entiers de $K$ ayant des polynômes minimaux stables sur $\mathbb{Q}$.

Maintenant et plus généralement on peut définir un polynôme générique du corps $K$ en prenant $\left\{w_{1}, \ldots, w_{n}\right\}$ une base quelconque de $K$ et en construisant le polynôme $F\left(u_{1}, \ldots, u_{n}, x\right)=\operatorname{Irr}\left(u_{1} w_{1}+\cdots+u_{n} w_{n}, L, x\right)$. Pour étudier sa stabilité, on peut choisir n'importe quelle base de $K$. En effet, soient $\left\{\theta_{1}, \ldots, \theta_{n}\right\}$ une autre base de $K, v_{1}, \ldots, v_{n}$ des variables algébriquement indépendantes sur $K, H=\mathbb{Q}\left(v_{1}, \ldots, v_{n}\right)$, et soient $\eta=v_{1} \theta_{1}+\cdots+v_{n} \theta_{n}$, $G\left(v_{1}, \ldots, v_{n}, x\right)=\operatorname{Irr}(\eta, H, x)$. On va vérifier qu'il y a équivalence entre la stabilité de $F$ sur $L$ et celle de $G$ sur $H$ :

Soit $M$ la matrice de passage de $\left\{\theta_{1}, \ldots, \theta_{n}\right\}$ à $\left\{w_{1}, \ldots, w_{n}\right\}$. Alors

$$
\begin{aligned}
\xi & =u_{1} w_{1}+\cdots+u_{n} w_{n} \\
& =\left(u_{1}, \ldots, u_{n}\right) \cdot\left(\begin{array}{c}
w_{1} \\
\vdots \\
w_{n}
\end{array}\right)=\left(u_{1}, \ldots, u_{n}\right) \cdot M \cdot\left(\begin{array}{c}
\theta_{1} \\
\vdots \\
\theta_{n}
\end{array}\right) \\
& =\left(v_{1}^{\prime}, \ldots, v_{n}^{\prime}\right) \cdot\left(\begin{array}{c}
\theta_{1} \\
\vdots \\
\theta_{n}
\end{array}\right)=v_{1}^{\prime} \theta_{1}+\cdots+v_{n}^{\prime} \theta_{n} .
\end{aligned}
$$

Cela montre que $F\left(u_{1}, \ldots, u_{n}, x\right)=G\left(v_{1}^{\prime}, \ldots, v_{n}^{\prime}, x\right)$. Or les $v_{i}^{\prime}$ sont linéaires en $u_{1}, \ldots, u_{n}$ et réciproquement ceci implique que $L=\mathbb{Q}\left(u_{1}, \ldots, u_{n}\right)=$ $\mathbb{Q}\left(v_{1}^{\prime}, \ldots, v_{n}^{\prime}\right)$ et ils sont algébriquement indépendants sur $\mathbb{Q}$; donc on peut prendre $v_{1}=v_{1}^{\prime}, \ldots, v_{n}=v_{n}^{\prime}$ et par suite $\xi=u_{1} w_{1}+\cdots+u_{n} w_{n}=v_{1} \theta_{1}+\cdots$ $+v_{n} \theta_{n}=\eta, F\left(u_{1}, \ldots, u_{n}, x\right)=G\left(v_{1}, \ldots, v_{n}, x\right)$. Ainsi on obtient l'équivalence du stabilité de $F$ et $G$ sur $L$.

Remerciements. Je tiens à remercier mon directeur de thèse M. Ayad pour les discussions ainsi que pour ses conseils qui m'ont permis de mener à terme ce travail. 


\section{Références}

[1] M. Ayad, Théorie de Galois, 122 exercices corrigés, niveau I, Ellipses, Paris, 1997.

[2] —, Théorie de Galois, 115 exercices corrigés, niveau II, Ellipses, Paris, 1997.

$[3]-$ - On irreducible polynomials over $\mathbb{Q}$ which are reducible over $\mathbb{F}_{p}$ for all $p$, preprint, 2004.

[4] M. Ayad and D. L. McQuillan, Irreducibility of the iterates of a quadratic polynomial over a field, Acta Arith. 93 (2000), 87-97.

[5] L. Danielson and B. Fein, On the irreducibility of the iterates of $x^{n}-b$, Proc. Amer. Math. Soc. 130 (2001), 1589-1596.

[6] G. Dumas, Sur quelques cas d'irréductibilité des polynômes à coefficients rationnels, J. Math. Pures Appl. (6) 2 (1906), 191-258.

[7] H. Hancock, Foundations of the Theory of Algebraic Numbers, Vol. 2, Mac Millan, New York, 1931.

[8] S. Lang, Algebra, Addison-Wesley, 1965.

[9] D. A. Marcus, Number Fields, Springer, 1977.

[10] R. W. K. Odoni, The Galois theory of iterates and composites of polynomials, Proc. London Math. Soc. 51 (1985), 385-414.

[11] N. G. Tschebotaröw, Grundzüge der Galois'schen Theorie, translated from Russian by H. Schwerdtfeger, Noordhoff, Groningen, 1950.

Université du Littoral

Côte d'Opale

50 rue Ferdinand Buisson

F-62228 Calais Cedex, France

E-mail: Ali.Nidal@lmpa.univ-littoral.fr

Reçu le 13.9.2004

et révisé le 10.3.2005 